\title{
The Fraser illusion: Complex figures
}

\author{
G. W. STUART and R. H. DAY \\ Monash University, Clayton, Victoria, Australia
}

\begin{abstract}
The cause of the Fraser illusion, which occurs when a line made up of tilted segments itself appears tilted, is examined further. In this series of experiments, we used figures that resembled the original Fraser illusion; they were more complex than those reported on in our previous paper (Stuart \& Day, 1988). The figures were used to explore two theories of the Fraser illusion further: that it is the result of interactions between orientation selective units, and that it is a consequence of the local, distributed processing of orientation. The presence of background elements like those used in the original illusion led to an increase in the strength of the illusion, but the shape of these elements had no differential effect on illusion strength. There was a differential effect of the background on the assimilative and contrast illusions, owing respectively to small and large tilts of the inducing elements. The illusion was markedly reduced at small visual angles when the background was absent, but it was only slightly affected when the background was present. All these findings are difficult to explain in terms of interactions between single units, either at the same or at different scales in the image. The effects of luminance contrast and isoluminance on the illusion were not consistent with either theory, but they indicated that researchers need to consider the role of figure-ground organization in this illusion.
\end{abstract}

The Fraser illusion is a compelling illusion of perceived orientation in which a line composed of tilted segments takes on the apparent orientation of those segments. Two versions of the illusion, the "LIFE' figure and the spiral illusion, are shown in Figure 1. In a previous paper (Stuart \& Day, 1988), we reported on a series of experiments carried out with simpler versions of the illusion, in which lines were made up of a series of tilted elements set at various angles to the slope of the line as a whole. Like Tyler and Nakayama (1984), we found that the illusion reversed at element angles greater than $18^{\circ}$. The same angular function was observed in the case of comparable Zöllner illusions, in which a plain black line was set against a series of short inducing elements. When these were made longer, to form more extensive backgrounds, the illusion reversed at smaller inducing orientations.

The generally accepted explanation of the Zöllner illusion is that it results from orientation-specific inhibition between cortical cells tuned to the same or to similar spatial locations (i.e., within $1^{\circ}$ for foveal vision; Oyama, 1975). It has been suggested (Adam, 1964; O'Toole \& Wenderoth, 1977) that the Fraser illusion is a reverse Zöllner illusion, in that orientation-specific interactions become facilitatory at small angles. There are a number of problems with this idea, the most important of which is a lack of clear psychophysical evidence for orientation-

The assistance of $\mathrm{V}$. Kohout and $\mathrm{J}$. Sack in the preparation of the experimental materials is gratefully acknowledged. We also thank Terry Bossomaier and Geoff Henry for helpful advice and discussion. Requests for reprints should be addressed to G. W. Stuart, Centre for Visual Sciences, Australian National University, GPO Box 475, Canberra, ACT 2061, Australia. specific facilitation in the Zöllner illusion, or in other angular distortions (Stuart \& Day, 1988).

Since to sustain orientation acuity it would seem important to maintain inhibition at all orientations, a more plausible theory is that of Tyler and Nakayama (1984), who proposed that the orientation-specific interactions responsible for the Fraser illusion occur between cells with different receptive field sizes. In this case, cells responsive to the orientation of the small elements are held to facilitate cells with larger receptive fields responsive to the global orientation of the line, producing a peak in activity away from the true orientation of the line. At larger element angles, the interactions become inhibitory, producing a reverse illusion.

A third theory of the illusion, proposed by Howard (1982) and elaborated by Stuart and Day (1988), is based on the premise that orientation is processed on a local basis, so that in foveal vision (or more exactly, in the case of receptive fields centered on the fovea) only lines between $6 \mathrm{~min}$ and $1^{\circ}$ of arc can be processed by a single cortical cell. There are both psychophysical evidence from subthreshold summation experiments (Bacon \& KingSmith, 1977; Thomas, 1978) and direct neurophysiological evidence from Area V1 of the monkey (Dow, Snyder, Vautin, \& Bauer, 1981; Poggio, 1972) to support this premise. According to this theory, the Fraser illusion reflects the fact that in the case of lines longer than $1^{\circ}$, global orientation perception is dependent on the integration of local information obtained from parts of the line. The Fraser illusion produces a faulty perception of global orientation because the "parts" of the line (the tilted elements) have a different orientation to the whole.

When we first outlined this theory, there was no direct physiological evidence relating to the hypothesized link- 

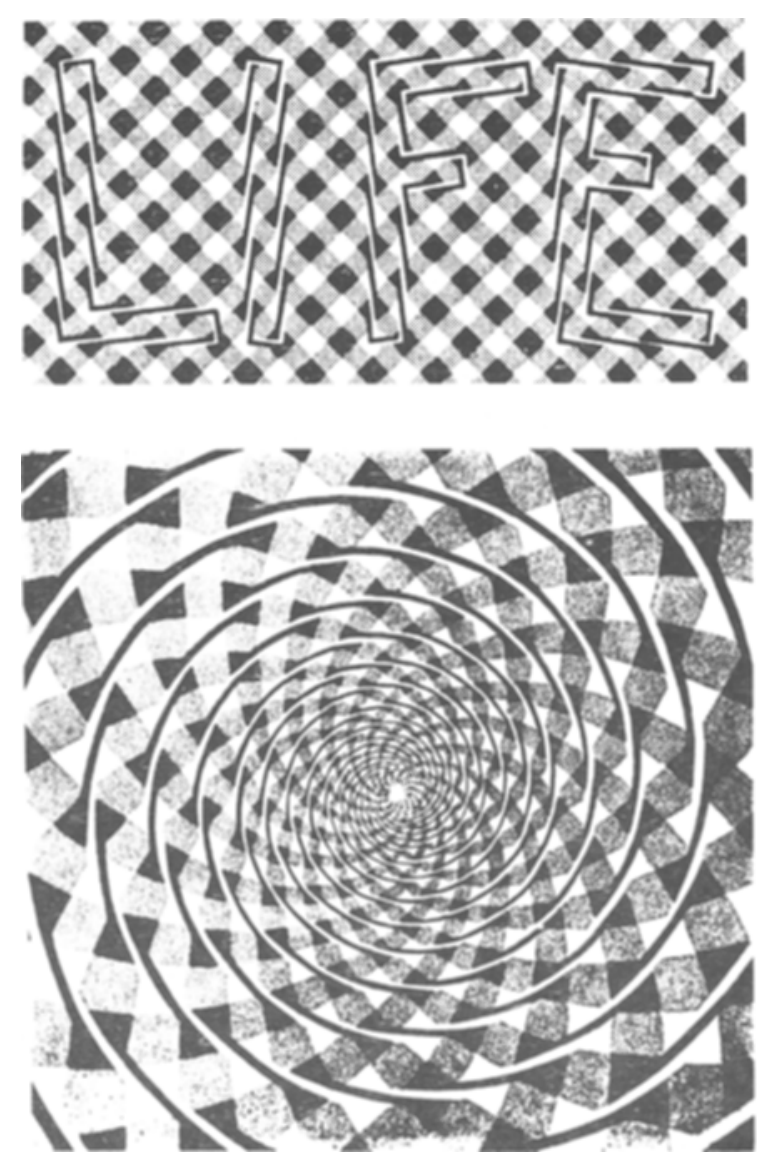

Figure 1. The "LIFE" and spiral forms of the Fraser illusion.

ing or integration mechanism. Recently, Eckhorn et al. (1988) and Gray, Konig, Engel, and Singer (1989) have described interactions between cortical cells that have many of the features required of such a linking mechanism. Using multiple-electrode recording techniques to record from cells in different cortical columns, Gray et al. found that cells in Area 17 of the cat with similar orientation preferences, but spatially separated receptive fields, displayed phase locking of oscillatory firing patterns. This phase locking occurred even when the cells had orientation preferences that differed by as much as $22^{\circ}$, and it was enhanced when both receptive fields were stimulated by a single long line. They concluded that phase locking serves to link transiently features in different parts of the visual field that may form the boundaries of objects. Eckhorn et al. (1988) reported that this linking of oscillatory activities occurred both within and between Areas 17 and 18 in the cat.

Because of the large tolerance shown by this linking mechanism with respect to the orientation of individual units, it would be capable of linking units responsive to parts of complex boundaries. While this tolerance confers the advantage of increased generality, it would mean that when the units are not collinear, as in the Fraser illusion, linking would still occur. This would produce a conflict between the orientation signaled by individual units and the spatial arrangement of their receptive fields, something that would not occur for real continuous boundaries. We suggest that, up to a point limited by the spatial arrangement of the individual units, the orientation signaled by the individual units makes a significant contribution to the perceived global orientation of a long boundary. Our previous results suggest this is maximal at $12^{\circ}$, but that it does not extend much beyond $18^{\circ}$, which is consistent with Gray et al.'s (1989) rough estimate.

The results of a previous set of experiments (Stuart \& Day, 1988) enabled us to rule out the first theory as an explanation of assimilation effects in both the Fraser and Zöllner illusions, for we found that local orientationspecific interactions were always inhibitory. However, we invoked lateral inhibition to explain the contrast effects seen at larger inducing angles in both of these illusions. The results of these experiments with simple line figures can be explained by Tyler and Nakayama's (1984) theory, since they proposed facilitatory interactions at small angles and inhibitory interactions at larger onesbut between cells with different receptive field sizes. In this sense, theirs is a local-to-global theory. As we outlined (Stuart \& Day, 1988), these results can also be explained in terms of the integration of information (which we now suggest is done by temporal linking of their activity) from local orientation analyzers. The two key differences between these theories are as follows: (1) Tyler and Nakayama (1984) suggest that global information is carried by single cells with large receptive fields, whereas we suggest that global information is coded cooperatively by a linked assembly of cells. (2) We maintain that this linking occurs only between cells with similar orientation preferences, and that the orientation contrast illusions seen at larger inducing angles are due to the influence of local distortions produced by local inhibitory interactions, prior to linking, rather than to the direct local-to-global inhibition suggested by Tyler and Nakayama (1984).

In this paper, we report on the use of more complex versions of the illusion that more closely represent the original figures of Fraser (1908), with which we tested these alternative theories further. With reference again to Figure 1, it can be seen that both illusions are made up of similar elements, which consist of a checkered background and a series of very salient figural elements that have the appearance of a "twisted cord" made up of black and white strands, since the elements making up the contours are at a different angle to the contours themselves. In the spiral form of the illusion (Figure 1B), the contours give a strong impression of a series of spirals, although close inspection reveals them to be a series of concentric circles. In the "LIFE" figure, the contours defining the letters appear to be tilted with respect to one another, even though they are all either parallel or orthogonal.

Fraser (1908) considered the critical feature of the illusions to be the tilted elements making up the distorted 
contours, since the "twisted cords" take on the orientation of those elements. He made a number of observations on the role of the checkered background in the complex forms of the illusion. In particular, he showed that the background alone does not induce an illusion. The tilted elements, when isolated from the background, as in Figure 1A of Stuart and Day (1988), do give rise to an illusion. This appears to be weaker than that present in the full version of the illusion.

A possible explanation for this is that in these more complex figures, the "twisted cords" are the result of figureground segregation. The cords are seen as separate from the checkered background, but at the level of the basic image there are no physical boundaries that have the same orientation as the cords themselves. Close inspection of Figure 1 reveals that no physical edges define the "twisted cords." In fact, the physical boundaries define a series of "directional elements" (Fraser, 1908), made up of triangles joined by tilted line elements. For one to perceive the "twisted cords," the triangles at the ends of the directional units must be seen as part of the checkered background, leaving the twisted cords figurally independent from that background. This may lead to a greater emphasis on the tilted elements, owing to the removal of other cues to the true orientation of the line that were present in the simple figures without any background used by Stuart and Day (1988).

Fraser reported a number of other observations of the "LIFE" figure that are relevant to this study. When letters not made up of twisted strands were tilted to match the orientation of those in Figure 1A, the required tilt was "slightly less" than that of the units of direction. So even in the strongest version of this illusion, the contours do not completely assimilate to the orientation of the elements. The illusion persisted at a wide range of visual angles, although no indication of any change in strength was given. The illusion was only eliminated at visual angles at which the resolution of the elements would become difficult. It also showed a horizontal-vertical anisotropy; when the "LIFE" figure was tilted by $90^{\circ}$, the longer elements of the letters appeared to be more tilted than they did when they were vertical. The illusion persisted when it was illuminated by a $1 / 3-\sec$ flash (ruling out a role for eye movements), and it was not diminished by steady fixation.

The spiral form of the illusion has been the subject of the only other extensive study of the Fraser illusion since Fraser's original report (Cowan, 1973). This figure is much more complicated than the "LIFE" figure, and it gives rise to effects additional to the tilt of straight lines produced in the simpler versions of the illusion. Fraser (1908) pointed out the strong impression of spiral forms in this figure, but he did not carry out a systematic study. He merely examined the same factors that were manipulated in the case of the "LIFE" figure. The spiral form of the illusion, like the "LIFE" figure, was unaffected by brief exposures or steady fixation. There were changes, however, with changes in visual angle. Fraser (1908) observed that when the size of the illusion was decreased, the inner circles of the figure became more easily confused with their immediate neighbors, producing an increase in the impression of spirals in this region of the figure.

Cowan (1973) set out to determine the relative importance of the various elements in the spiral form of the illusion, noting that there are a number of spiral components to this figure. First, there are the directional elements themselves, which are very similar to the compound directional elements in the "LIFE" figure. Even though the twisted cords themselves trace out circular paths, each of the directional elements forms part of a spiral, and it is the spiral form that is perceived. Second, there are spiral components in the checkered background itself. In fact, there are two sets of oppositely directed spirals. Third, if the directional elements are grouped with those in neighboring cords, these groupings would also follow an underlying spiral path.

By manipulating these elements of the spiral figure in various ways, Cowan (1973) was able to determine their role in the formation of the illusion. With one exception, Cowan found that no matter how the other components of the illusion were manipulated, it was the directional elements of the twisted cords that determined the direction of the illusion. The exception was the case in which, at very small visual angles, physical groupings of adjacent elements (peculiar to Cowan's version of the illusion) determined that direction. Note that at this angle, the tilted line segments of the directional elements were still within the range of normal visual acuity, and so it seems that there is an optimal size at which the directional elements are most effective.

Although Cowan (1973) demonstrated the importance of directional elements in the spiral form of the illusion, he did not establish a basis for this in terms of normal visual processing. In fact, he considered that it might be due to facilitation between orientation detectors, and, like Tyler and Nakayama (1984), he produced a reverse version of the "LIFE" figure by changing the angle of the directional elements. In the experiments described here, the relative merits of theories based on orientation-specific interactions, and the "orientation integration" theory, based on the local, distributed processing of orientation information, were explored further.

\section{GENERAL METHOD}

The general method, described in detail in Stuart and Day (1988), can be briefly summarized as follows:

\section{Subjects \\ The subjects were paid volunteers between the ages of 17 and 30 who had at least $20 / 20$ acuity for near vision.}

\section{Apparatus}

The experimental stimuli were photographs mounted on cardboard disks $21.3 \mathrm{~cm}$ in diameter. An adjustable black line was made on the underside of a clear Perspex disk. When this clear disk was placed on top of the photographs, the adjustable line and the experimental stimulus were in the same plane. The adjustable line, which was $4 \mathrm{~cm}$ long, with one end at the center of the display, 
could then be adjusted through rotation of the Perspex disk until the line appeared at right angles to the stimulus lines. These were usually $8 \mathrm{~cm}$ long, tilted at $45^{\circ}$ counterclockwise, $3 \mathrm{~cm}$ away from the center of the display. A chinrest held the viewing distance at $64 \mathrm{~cm}$, so that the stimulus lines subtended a visual angle of $7.2^{\circ}$.

\section{Statistical Analysis}

Where appropriate, the multivariate analogue to repeated measures analysis of variance (ANOVA) was used. These $F$ values are identified by the subscript $M$.

\section{EXPERIMENT 1}

In this experiment, two basic issues were addressed: How much stronger is the illusion with the background present relative to simple line versions of the illusion, and does the form of the background have any influence on the strength of the illusion? The latter question was posed by Robinson (1972), but here it was given theoretical weight as a test of the competing theories of the Fraser illusion. Specifically, if the triangular components of Fraser's "directional elements" add to the lateral facilitatory interactions in a way that other background elements would not be predicted to, this would provide support for the lateral facilitation theory. If all forms of background are equally effective, this would indicate that one of the two other theories was correct. Tyler and Nakayama (1984) suggested that the stronger illusion in the complex illusions is "probably due to weak stimulation of cells with large receptive fields ... as a result of the presence of light and dark patches in the summation region of such receptive fields" (p. 538). If this is the case, the detailed form of the background would not be critical. The same prediction follows if local orientation information is the critical determinant of the illusion, and if the background increases the dependence on misleading local information by removing competing local orientation cues present in the simpler versions of the illusion.

\section{Method}

Subjects. Twenty subjects, 14 females and 6 males, took part in Experiment 1.

Apparatus. The apparatus was the same in all respects to that described earlier (Stuart \& Day, 1988).

Materials. Only five experimental lines, shown in Figure 2, were used in this experiment. All were versions of the Fraser illusion with eight elements set at $8^{\circ}$, with the exception of the control line. One was a simple line version of the illusion, like those used in the previous experiments. Three of the others were single-line versions of the "LIFE" figure with minimal background-that is, a series of directional elements arranged in a line to give the impression of a "twisted cord" against a background of a series of shapes. In one of these, shown in Figure 2, the directional elements were the same as they were in the original Fraser illusion, and they produced the impression of a series of squares oriented along their oblique axes. In the other two figures, the directional elements were partly formed from circles, to give a background of a series of circles, or from rectangles, which gives the impression of a series of squares oriented in the same direction as the tilted elements. The idea behind this was to create background elements whose edges met those of the oblique sections at angles that would not create any additional lateral interactions-that is, they were parallel or or-

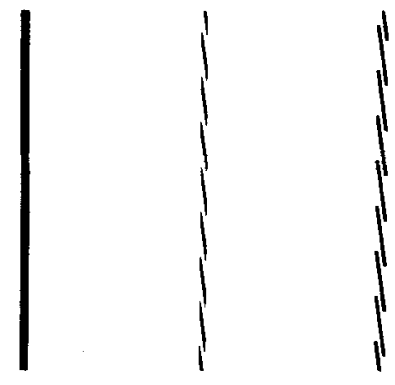

CONTROL LINE ONLY CLUBBED

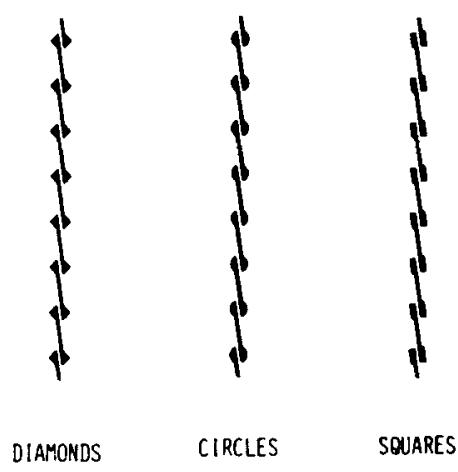

Figure 2. Stimulus figures used in Experiment 1.

thogonal to them, or they contained a whole range of orientations as in the case of the circular elements.

The fifth experimental line was a version of the illusion that had no background elements, but in which the elements were not smoothed off to make a "barber's pole" as in previous experiments. This figure is comparable to the illusions without background that were designed by Robinson (1972). In this version of the illusion, the vertical edges present in the simple version are removed, which may lead to an increased illusion. This is done without the introduction of any additional local interactions, as may be the case when background elements are used. In addition, this version should not significantly weaken the response of cells responsive to the global orientation of the twisted cords.

Design. Each subject made matching adjustments to all of the illusory lines. A Latin square design was used, with each line presented four times in each of the six possible serial positions (including the control line). This required four complete Latin squares, each based on a different initial serial order.

Procedure. This was exactly the same as that described in the General Method section of Stuart and Day (1988).

\section{Results and Discussion}

The results of Experiment 1 are shown in Figure 3. A one-way ANOVA was carried out to see if there was any overall difference between the strengths of the various illusion figures. A significant overall effect was obtained $\left[F_{M}(4,20)=17.06, p<.0001\right]$. Because of the heterogeneity of variance between conditions, paired comparisons were carried out with Bonferroni-adjusted paired $t$ tests. These showed that the illusions with background were not significantly different from each other, but that 


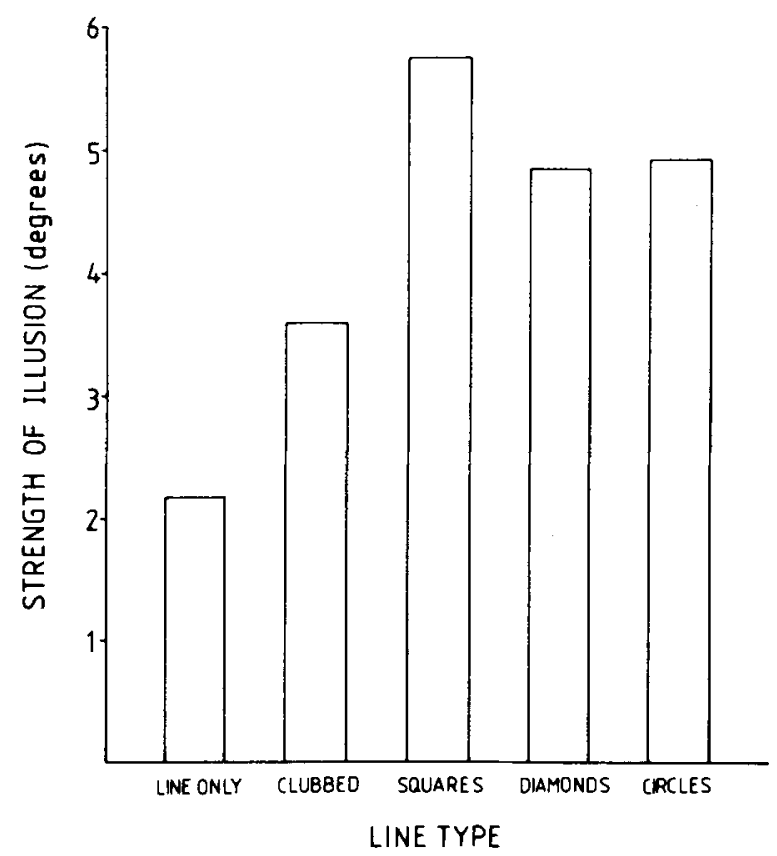

Figure 3. Results of Experiment 1. The strength of each illusion is expressed as the difference in degrees from the apparent orientation of the control line.

they were stronger than the version of the illusion with clubbed elements, which in turn was stronger than the version with no background elements that had a line-like appearance. The figure with diamond elements just failed to reach the adjusted significance level.

The data from Experiment 1 support the view that additional local orientation-specific interactions are not involved in the stronger illusions in the figures with background elements, since the form of the background did not affect the illusion. Tyler and Nakayama's (1984) proposition that the background elements weaken the response of cells responsive to the global orientation of the twisted cords is consistent with this finding. However, it seems that the figure with clubbed elements would not produce much more contrast cancellation than would the line-like figure, so it is difficult to explain this finding in such terms. We suggest the following, more plausible, explanation. In the simple line figures, the vertical edges of the elements are the same distance apart, but they are more collinear than the oblique edges. (In fact, they tilt in the opposite direction, because of local contrast effects.) So although the oblique edges may be linked into a distributed representation, the vertical edges would also form a competing linkage, or perhaps the two representations would be combined. In either case, the contribution of the oblique edges would be diluted. When the background elements are present, only the oblique edges contribute to the distributed representation, leading to a stronger illusion. When the elements are clubbed, the edges are removed, but the line terminators may contribute some orientation information (perhaps via a linking of the activity of hypercomplex cells), leading to an intermediate illusion.

\section{EXPERIMENT 2}

The aim in Experiment 2 was to explore further the question of how the background in the more complex forms of the Fraser illusion produces a more powerful illusion. It has already been suggested that the background elements act by removing competing local cues to the orientation of the twisted cord that are present in the edges of the elements in the simple line form. Alternatively, Tyler and Nakayama (1984) suggested that the background reduces the response of cells sensitive to the global orientation of the line, making them more susceptible to the influence of cells tuned to the smaller tilted elements.

One way to test these hypotheses further would be to ascertain what effect the background has on Fraser-type illusions with different element angles, particularly at larger angles where a contrast illusion is present in the simple line versions. If Tyler and Nakayama's (1984) suggestion is correct, then we would argue that the background should increase the strength of both assimilation and contrast illusions. On the other hand, we have argued that a distributed representation of a line should only consist of contributions from local orientation analyzers with similar orientation preferences. We explained contrast illusions in the simple versions of the Fraser illusion in terms of local distortions produced by orientation-specific inhibition prior to linking. Because the background eliminates the local edges that contribute to the contrast illusions, we predict that for these illusions the effect should be reduced or eliminated.

To test these hypotheses, it was necessary to design versions of the illusion in which the form and number of the background elements was preserved but in which the angle of the internal elements covered a wide range of angles. With a checkered background, the angle of the elements is constrained by the thickness of the cord and the size of the checkered background. Because the form of the background elements seemed to make little difference to the illusion in Experiment 1, radically different background elements were used so that these could be kept constant as the orientation of the directional elements was changed. These versions of the illusion will be described in greater detail in the Materials section.

\section{Method}

Subjects. Twenty subjects, 11 males and 9 females, took part in the experiment.

Apparatus. The apparatus was exactly the same as that used in Experiment 1.

Materials. The materials used in Experiment 2 are shown in Figure 4. It is apparent at first glance that these are radically different from the forms of the Fraser illusion that have been used so far. These differences-principally the thickness of the line $(3 \mathrm{~mm}$ rather than $2 \mathrm{~mm}$ ), the number of elements (16 rather than 8 ), and the form of the background elements-were dictated by the need to satisfy the following conditions: (1) that the number and form of the background elements should remain constant; (2) that the thick- 


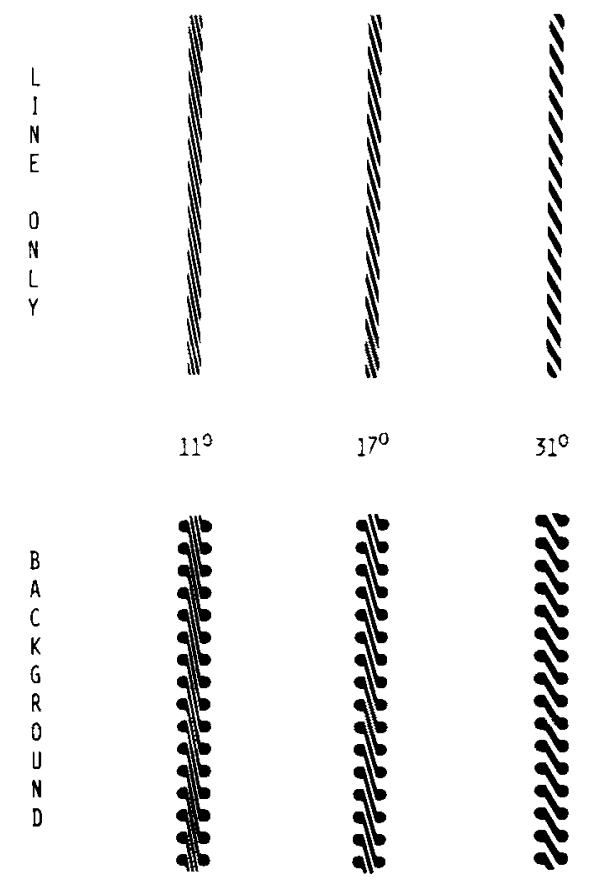

Figure 4. Stimulus figures used in Experiment 2. These lines were exactly the same, except for the presence of an identical set of background elements in the stimuli on the bottom row.

ness of the line should also remain constant; and (3) that the angles used should be those expected to produce a strong positive, a neutral, and a strong negative illusion.

These conditions were satisfied through the use of figures with the background elements and the cord thickness described above and with the construction of directional elements whose tilted segments connected together parts of these background elements. Through the connection of elements that were immediately adjacent, or were separated by one or two intervening elements (see Figure 4), it was possible to obtain element angles of $11^{\circ}, 17^{\circ}$, and $31^{\circ}$, which, on the basis of the results of Stuart and Day (1988), were expected to satisfy the third condition that there should be a positive, a negative, and a neutral illusion-although the major changes in other features of the illusion meant that this was by no means certain. The three different illusions were constructed both with and without background elements, to give a total of six experimental figures. A control line of the same length and thickness as those of the "cords" in these figures was also included.

Design. A simple two-factor repeated measures design was used, one factor being the angle of the elements, the other the presence or absence of background elements. All subjects made matching adjustments to the six experimental lines and the control line. The figures were presented in a strictly random order.

Procedure. The procedure was exactly the same as that in Experiment 1.

\section{Results and Discussion}

The results of Experiment 2 are shown in Figure 5. They were analyzed with a two-way repeated measures ANOVA. This showed that there was a main effect of the angle of the directional elements, as had been expected $\left[F_{M}(2,18)=93.24, p<.0001\right]$. There was also a main effect of the presence of background, the figures with background showing overall more positive illusion $[F(1,19)=$
$15.09, p<.001$ ]. In addition, there was a significant interaction between the two factors $\left[F_{M}(2,18)=8.52\right.$, $p<.01$ ]. This seemed to be due mainly to a lack of difference between the illusions with a $17^{\circ}$ element angle.

The results indicate that whereas the positive illusion was increased by the presence of a series of background elements, the negative illusion was reduced in strength by the presence of these elements. It appears that once again the conclusions of earlier experiments were supported, in that the assimilation illusion is a direct illusory effect that is increased in strength when the background is present, because of the absence of competing local orientation cues. The presence of an interaction effect, with a smaller difference between the two angular functions at $17^{\circ}$, is evidence that the assimilation and contrast illusions have a different basis and operate over a different angular range. The results indicate that the direct contribution of the directional elements is stronger at smaller angles but operates over a narrow range. The contrast component due to local inhibition operates over a wider range, peaking at much larger angles. At $17^{\circ}$, both components are relatively weak, producing only a slight effect, which is not greatly affected by the presence of the background. At $11^{\circ}$, there is a strong direct effect that is enhanced by the removal of competing local cues. At $31^{\circ}$, there is a strong contrast effect, which depends on local inhibitory interactions that are reduced by the presence of the background.

It is also necessary to explain why the contrast illusions were not completely eliminated by the background. This was probably because of the presence of subjective contours defined by the junctions between the horizontal and tilted segments of directional elements in the complex forms of the illusion. It is known that subjective contours or rows of dots can be distorted in orientation illusions (Coren, 1970; Smith \& Over, 1977), and that there are

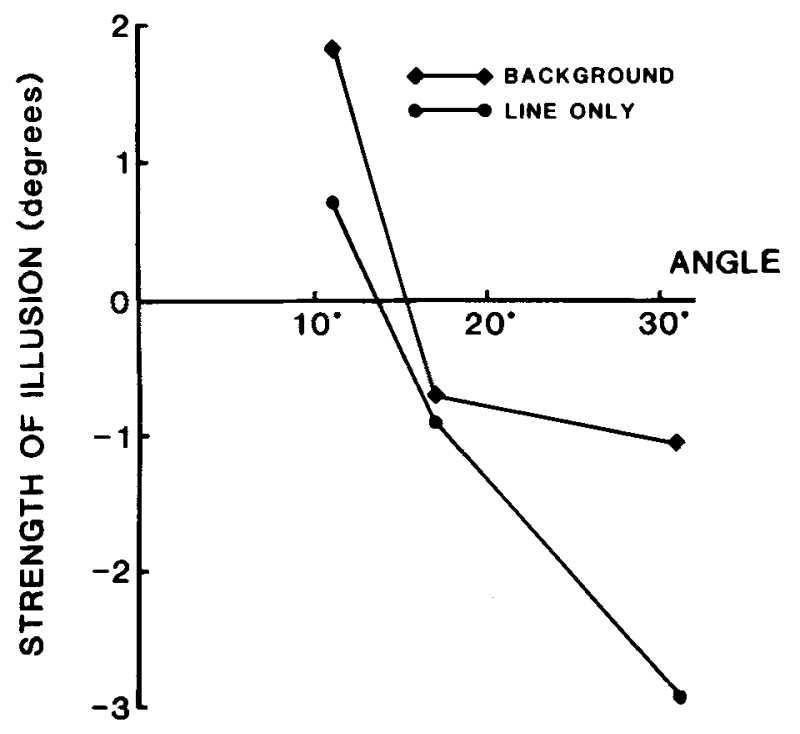

Figure 5. Results of Experiment 2, showing the differential effect of the background on the assimilation and contrast illusions. 
cells in Area V2 of the visual cortex that respond preferentially to subjective contours (von der Heydt, Peterhans, \& Baumgartner, 1984) or to rows of dots (Peterhans \& von der Heydt, in press). It has now been directly demonstrated that orientation-specific inhibition occurs between cells responsive to real and subjective contours (von der Heydt \& Peterhans, 1989). This is almost certainly the basis of the residual contrast illusions in the figures with background elements.

\section{EXPERIMENT 3}

The aim in Experiment 3 was to examine the behavior of the Fraser illusion at different visual angles. This was of interest for a number of reasons. Perhaps the most important was that it would provide a direct test of the theory of Tyler and Nakayama (1984) that the illusion results from interactions between orientation detectors tuned to the orientation of the elements and those tuned to the orientation of the line as a whole. If that theory was correct, it might be argued that the illusion should be strongest at smaller visual angles, where the whole line falls within the upper range of receptive field size of orientation detectors.

Another reason was to compare the behavior of the simple line forms of the illusion to the complex form with background elements. Since the view has been put forward that the complex form of the illusion owes its greater strength to a masking of other orientation cues, it was thought that this form of the illusion would not change markedly with changes in visual angle size. On the other hand, it was thought that when the simple form of the illusion is viewed with most of it lying in near-foveal areas of the visual field, the orientation of the whole figure, or large parts of it, could be coded by the largest local analyzers, leading to a decrease in illusion strength. Thus the integration theory would seem to predict the opposite of Tyler and Nakayama's (1984) theory with regard to the simple form of the illusion. It is difficult to derive specific predictions from the latter theory with regard to the complex form of the Fraser illusion, apart from an increase in illusion strength in the complex figures.

\section{Method}

Subjects. Twenty persons, 9 males and 11 females, participated in the experiment.

Apparatus. To allow the use of larger stimulus lines in this experiment, a larger version of the apparatus was constructed. It was similar in nearly all respects to the apparatus used in previous experiments, except that the disks on which the photographs were mounted were $30 \mathrm{~cm}$ in diameter. The inner diameter of the annulus that masked the lines used to measure the position of the clear disk was $29 \mathrm{~cm}$. The chinrest was lowered so that on the average the subjects' eyes were $48 \mathrm{~cm}$ from the display.

Materials. The lines in this experiment were the $8^{\circ}$ Fraser illusions, both with and without background, and a control line. These were presented at different visual angle sizes, as can be seen in Figure 6. The visual angle changes were achieved through the magnification of all aspects of the display precisely, so that the effect was the same as that which would be obtained through changing the viewing distance from the display. Versions of the simple, com-

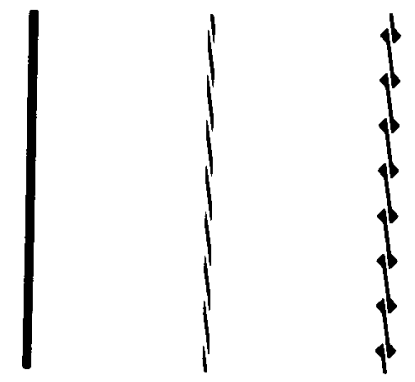

CONTROL LINE ONLY BACKGROUND

PRESENTED AT FOUR VISUAL ANGLES:

1. $2.4^{\circ}$ ( 1 iN 24)

2. $4.8^{\circ} \quad(1 \mathrm{IN} 12)$

3. $9.5^{\circ}$ ( 1 iN 6$)$

4. $18.9^{\circ} \quad(1$ iN 3$)$

Figure 6. Stimulus figures used in Experiment 3. A control line, a simple line version of the illusion, and a version set against background elements were presented at the visual angles indicated at the bottom of the figure.

plex, and control lines were made, which were $2,4,8$, and $16 \mathrm{~cm}$ in length. As mentioned in the last section, the chinrest was lowered so that the visual angles subtended by these lines were $2.4^{\circ}, 4.8^{\circ}$, $9.5^{\circ}$, and $18.9^{\circ}$. Recall that in the experiments reported thus far, the illusion lines were $7.2^{\circ}$ of visual angle in length. All the other dimensions of the display - that is, the distance and orientation of the lines relative to the center of the display, and the length and thickness of the adjustable matching lines-were varied in the same ratios. The adjustable matching lines were made of black adhesive tape of the appropriate width.

Design. A two-factor design was used, the factors being type of illusion (simple or complex) and visual angle size. Both factors were subjected to repeated measures, with all subjects making matching adjustments to all the illusion lines and the four control lines. The lines were presented in a strictly random order.

Procedure. The procedure was exactly the same as that in Experiments 1 and 2 .

\section{Results and Discussion}

A two-way repeated measures analysis of variance was used to analyze the results, which are shown in Figure 7. All the trends apparent in this graph were significant. There was a significant main effect of visual angle $\left[F_{M}(3,17)=4.47, p<.05\right]$. The larger visual angles, in general, produced greater illusions. The complex figures once again produced a significantly greater illusion on the average $[F(1,19)=106.04, p<.0001]$. It appeared that the simple figures were much more sensitive to changes in visual angle size than the complex figures were, although this interaction just failed to reach statistical significance $[F(3,57)=2.16$, n.s.]. This result is probably not particularly important, because it does not take into account the fact that the illusion without background started from a baseline near zero. Proportionately, 


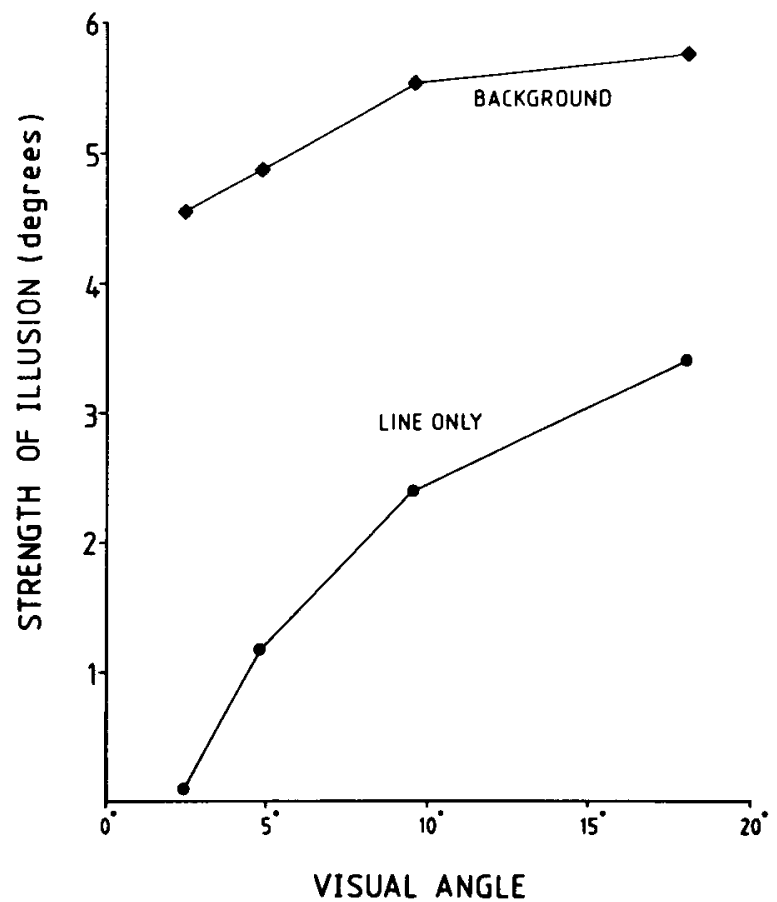

Figure 7. Results of Experiment 3, showing how the simple version of the illusion was markedly affected by its overall size, in contrast to the version with background elements.

the increase in illusion was much greater as visual angle increased, even if the absolute increments were similar.

These results are consistent with the idea that the Fraser illusion results from the integration of local orientation information in the perception of the tilt of larger objects. In the case of the simple form of the illusion, the orientation illusion was reduced at smaller visual angles, where the entire stimulus, or at least large parts of it, could be processed by single orientation-selective cells. It is difficult to see how the theory of Tyler and Nakayama (1984) can explain this result, particularly since in the complex version of the illusion there is still a strong effect. Note that the simple version of the illusion never reached the strength of the complex version. This indicates that even at the largest visual angle the veridical information present in the vertical edges of directional elements is used, and it counteracts the misleading information contained in the directional elements.

The behavior of the complex forms of the illusion is also consistent with the orientation integration theory. If the background removes competing local cues, as was established in Experiment 2, then these cannot be used, even at small visual angles, as they appear to be used in the case of the simple forms. Thus, the visual system depends even more on the directional elements in order to establish the orientation of the "twisted cord" in the Fraser illusion. At the largest visual angle used, the strength of the complex version of the illusion approaches the almost total assimilation that was claimed by Fraser (1908) and Cowan
(1973) for the most complex versions of the illusion, such as the "LIFE" figure and the spiral form. This indicates that in this version of the illusion, the cues to the true orientation of the line have been almost totally removed.

\section{EXPERIMENT 4}

The primary aim of this experiment was to provide a further test of the hypothesis that the simple and complex versions of the Fraser illusion reflect the operation of the same visual information processes in relation to orientation. One of the most important features of the illusions that are thought to result from orientation-specific inhibition is their dependence on luminance contrast (Davidoff, 1973; O'Toole, 1979; Oyama, 1975; Parker, 1974; Stuart \& Day, 1980; Wallace, 1975). Wallace (1975), who showed that the strength of the Zöllner illusion was a function of the log contrast of the lines making up the illusion, related this to the increase in the firing rate of cortical cells that accompanied increases in luminance contrast.

If the Fraser illusion is a result of orientation-specific interactions, it should also display some variations in strength as contrast changes. In addition, if the simple form of the illusion reflects such interactions, but if the more complex figures are the result of some higher order process, their response to changes in contrast might be different. It was by no means certain how these assimilative illusions would react to changes in contrast, because they had not been used in any previous studies. Fraser (1908) looked only at changes in the contrast between the black and the white "directional elements" in the full version of the illusion. If the model of O'Toole and Wenderoth (1977) for facilitation in assimilation illusions is correct, a reduction in the spread of inhibition associated with reduced luminance contrast might be expected to promote facilitation at small angles. This is because a decrease in inhibition produces broader population response functions, so the change from facilitation to contrast would occur at greater angles (see the original articles by Blakemore, Carpenter, and Georgeson [1970] and by O'Toole and Wenderoth [1977]). It is not clear how changes in contrast would affect the interactions specified in the model of Tyler and Nakayama (1984).

\section{Method}

Subjects. There were 20 subjects, 12 males and 8 females.

Apparatus. The apparatus was exactly the same as that used in Experiments 1 and 2.

Materials. The stimulus lines are shown in Figure 8. Due to the difficulty of producing low-contrast versions of the illusions, and since the main concern was the behavior of the simple and complex versions of the positive Fraser illusion, only one element angle, $8^{\circ}$, was used. It was also decided that both solid and outline versions of the illusions should be used to provide a link with previous work on orientation illusions, most of which had involved figures composed of lines rather than the edges of solid elements.

Fine lines, even of a very low objective contrast, show considerable subjective contrast enhancement. It was decided that the lines used should all be of equal subjective contrast. Thus the lowest pos- 


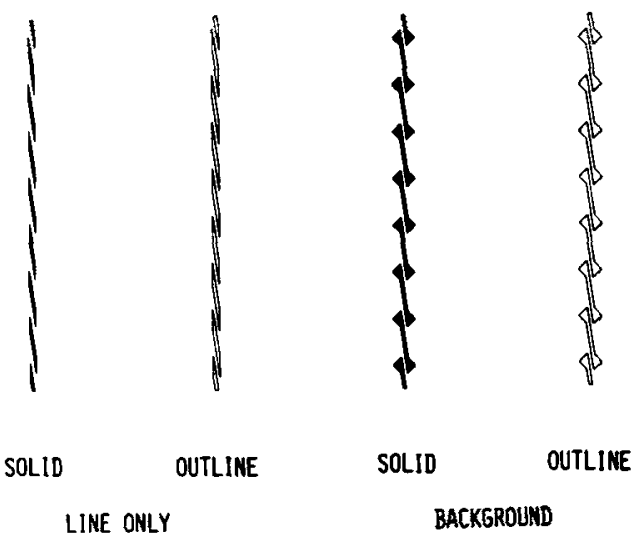

PRESENTED AT THO CONTRASTS: 1. HIGH (BLACK ON WHITE) 2.LOW (LIGHT GREY ON WIITE)

Figure 8. Stimulus figures used in Experiment 4. Outline and solid versions of both simple and complex illusions were presented at high and low contrasts.

sible contrast was effectively determined by the subjective appearance of the fine lines. At very low contrasts, during normal black and white photographic development, a certain amount of unevenness in print contrast results. Consequently, the low contrast used in Experiment 4 was determined by the lowest contrast at which even prints of the outline figures could be produced.

The following procedure was used. The first figure to be produced was the low-contrast control line. This was carefully matched to a density corresponding to $\mathbf{8 . 0}$ on the Munsell scale, which has an absolute reflectance of $57.56 \%$ (Judd, 1951). The density of the white background was 9.5 on the Munsell scale, an absolute reflectance of $87.66 \%$. The resulting contrast was .21 , using the following formula:

$$
C=\frac{I_{\mathrm{Max}}-I_{\mathrm{Min}}}{I_{\mathrm{Max}}+I_{\mathrm{Min}}} .
$$

The level of illumination was $700 \mathrm{~lx}$.

Next, many low-contrast versions of the other figures were produced at slightly different exposures. Two raters (G.S. and L.A.) compared these to the control under the same viewing conditions that had been used in previous experiments and selected a photograph of each of the remaining figures that was not noticeably different in subjective contrast from the control. It should be noted, however, that many of the figures were very close in contrast to each other and to the control line, so that sometimes the choice of a particular figure was somewhat arbitrary. The obvious advantage of this was that it ensured that the low-contrast figures were all very close in contrast to each other, despite the fact that it was impossible to use more sophisticated psychophysical methods.

With the same procedure, a low-contrast comparison line was also produced; it was fixed to the underside of a second clear Perspex disk with transparent gum. Thus a total of 10 lines were used in the experiment-the four illusion figures and the control line-all of which were produced at both high and low subjective contrasts.

Design. The experimental design included four factors, with repeated measures on three of them. The first two factors were the type and form of figure-that is, solid or outline versions of the simple and complex Fraser figures, plus a control line. The third factor was the contrast of the figures. All subjects made matching adjustments of all 10 figures, including both the high- and the low- contrast control lines. The fourth, non-repeated measures factor, was the contrast of the adjustable matching line. Half the subjects used the low-contrast comparison line; the other half, the highcontrast line. The various figures were presented in strictly random order.

Procedure. The procedure was identical to that in Experiments 1,2 , and 3 .

\section{Results and Discussion}

The first stage in the analysis was carried out to establish if there were any systematic variations in matching adjustments associated with the use of the different contrast control and comparison lines. A $2 \times 2$ ANOVA procedure was used to test this. Only the data from the four possible combinations of control and comparison lines were used. There were no significant main effects or interactions, indicating that contrast of control line was not a source of variation. In the remaining analyses, the control line of the appropriate contrast was used as the baseline for the illusion figures.

On this basis, a $2 \times 2 \times 2 \times 2$ four-way ANOVA was carried out. The results of the experiment, collapsed according to this design, are shown in Figure 9. There was no

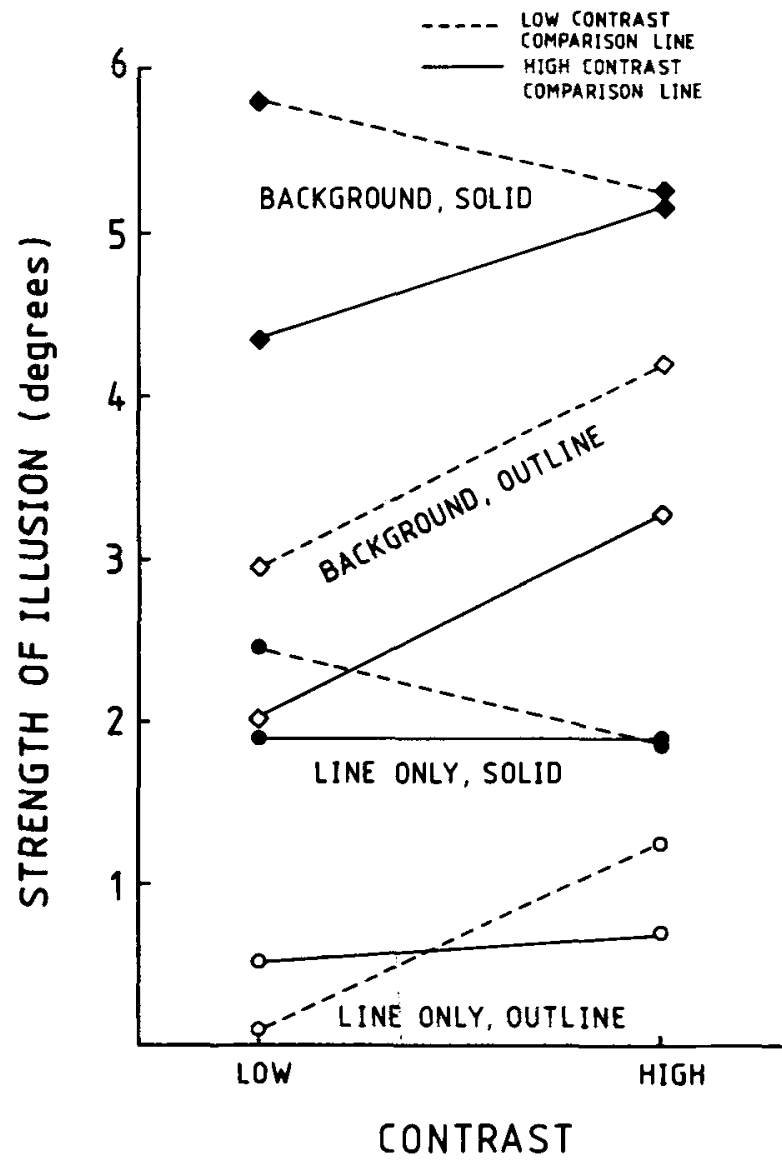

Figure 9. Results of Experiment 4. Solid symbols are used for solid illusions, outline symbols for the outline illusions. Diamonds represent the complex illusions; circles, simple line illusions. These were measured with either a high-contrast (solid lines) or a low-contrast (broken lines) comparison line. 
significant main effect of the contrast of the comparison line, but there was one significant interaction-between the form (outline vs. solid) and contrast of the illusions and the contrast of the comparison line $[F(1,18)=5.19$, $p<.05]$. This result reflects the finding that the lines without any background elements were not affected by the contrast of the comparison line, but the lines with background elements showed an increased illusion when the low-contrast comparison line was used.

This result was unexpected. It may have been due simply to subject variation, there being only 10 subjects in each group. Another possibility is that the high-contrast control line is easier to see in peripheral vision, enabling the subject to view the illusory line in more central vision, leading to increased orientation acuity and therefore decreased illusion.

The remaining three factors were those of greatest theoretical interest. As expected, a significant main effect was associated with the type of illusion. The complex figures, as expected, produced a much more positive orientation illusion than did the simple figures $[F(1,19)=$ $107.74, p<.0001$ ], in accord with the results of the previous three experiments. The solid figures also produced a greater average illusion than did the outline figures $[F(1,19)=35.25, p<.0001]$. This may be due, as was thought to be the case at least in the simple figures used in the previous paper (Stuart \& Day, 1988), to the different Fourier composition of solid and outline figures.

There was no significant main effect of contrast, and the only other significant effect was an interaction between contrast and the form of illusion $[F(1,18)=10.79, p<$ $.01]$. It seems that the solid figures were not affected by a reduction in contrast, but that the outline figures were considerably weakened in illusion strength. Both simple and complex figures were affected in the same way, since there were no higher order interactions.

This finding is not an obvious consequence of any of the theories we have considered. An interesting possibility is that the result represents variation in the strength of separation of "figure" (twisted cord) from " ground," an explanation used by Fraser (1908) himself. Although in the case of the simple figures there is no background for the elements to stand out from, some sort of grouping process must be involved for the "twisted cord," rather than a series of disconnected elements, to be seen.

\section{EXPERIMENT 5}

Experiment 5, the final one in the series, was carried out to test the claim of Gregory (1977) that the Fraser illusion is destroyed when the borders defining it are defined only by color contrast, the subjective brightness across the border being equalized. This was of interest for two reasons. First, if this is true, it could be argued that decreasing the luminance contrast affects lateral inhibitory processes, thus implicating them in the illusion. Second, Gregory (1977) claimed that it was the higher order form-perception processes operating in the illusion that were destroyed by isoluminance, in support of his theory that only the luminance channels contribute to higher order form perception.

There is a serious flaw in the argument that Gregory (1977) put forth to support the latter theory. He says of the spiral form of the Fraser illusion:

This lost its spiral form. It became extremely confusing and was difficult for observers to describe. Form perception was markedly disturbed and regions would fade. The effect was dramatic. (p. 117)

Although Gregory was not very specific about his definition of form perception, later researchers such as Ware (1981) have taken him literally and argued that higher order form processes of the Gestalt type are disturbed when a visual stimulus is composed of isoluminant color borders. Although Gregory (1977) claimed that the disturbances were not due to peripheral effects such as loss of acuity, his report that regions of the figure faded is not consistent with his own argument. If the observer can see only parts of the figure, it is not surprising that he or she should find it difficult to say whether it has a spiral or a circular form. The aim in Experiment 5 was to measure the strength of the Fraser illusion when it is composed of isoluminant color borders, using a single-line version of the illusion that subtended a visual angle of $7.2^{\circ}$, as in most of the earlier experiments in this study. It was hoped that this, as well as the fact that there was no set fixation point, would prevent the problem of parts of the figure "fading" (Livingstone \& Hubel, 1987).

\section{Method}

Subjects. Twenty persons, 10 male and 10 female, participated in the experiment.

Apparatus. Certain modifications had to be made to the apparatus. The technique was based on Gregory's (1977) method of producing isoluminant contours by simultaneous front and back projection. The original apparatus, which was made of white translucent Perspex, was added to in the following way. A mirror was placed at $45^{\circ}$ underneath the apparatus to direct the light from a slide projector containing a green (No. 57) Kodak Wratten filter directly onto the base of the apparatus. The stimulus lines were cut out of white single-weight photographic paper and glued to white Perspex disks. Thus, where the paper was cut out, those parts of the stimulus appeared green. The luminance of the green light passing through the Perspex was $3.3 \mathrm{~cd} / \mathrm{m}^{2}$. Above the apparatus was a second projector containing a red (No. 26) Wratten filter, and the light from this source was directed down onto the top of the apparatus. This illuminated the remaining part of the stimulus in red. The intensity of the light coming from the second projector could be varied by means of a Variac voltage regulator.

Materials. As mentioned above, the stimulus lines were cut out of single-weight photographic paper and then mounted on white Perspex disks. The figures were photographically produced in outline form at a very faint contrast, and then the appropriate sections were cut out with a surgical scalpel and the aid of a metal ruler. It proved difficult to do this with sufficient accuracy in the case of the simple form of the illusion, since the lines intersect at the small angle of $8^{\circ}$. However, with great care, it was possible to produce an acceptable version of the complex form of the illusion. Thus only four experimental stimuli were used in the experimentblack and white and isoluminant forms of the complex form of the Fraser illusions and of the control line. The luminance reflected 
from the white regions of the black and white figures was the same as that of the green sections of the isoluminant figures. A black adjustable matching line was used to measure the apparent orientation of all the lines.

Design. The design consisted of a simple comparison of the strength of the two illusions relative to their respective controls. The order of presentation of the figures was completely counterbalanced, using five Latin squares with different initial orders.

Procedure. The procedure was very similar to that in the previous experiments, except that it was necessary to find a setting for the voltage of the upper projector that represented isoluminance for each subject. The subjects adjusted the brightness of the red background until it matched that of the green illusion or the control. To aid them in this task, their attention was drawn to the fact that as the point of isoluminance was passed during adjustment there was a pronounced flicker (the brightness seemed to "jump" from illusion to the background and vice versa), even if the rate of luminance change was rather slow. They were encouraged to make several adjustments to either side of this point before making their final adjustment. This was done four times, and the average voltage was used as the point of isoluminance. Again this method was not very sophisticated psychophysically, but it should give a value very close to subjective isolunfinance. It is based on the perception of flicker, although not in the same way as the flicker-fusion technique, which because of the small size of the stimuli was not used. Nonetheless, it was a more sophisticated technique than that used by Gregory (1977), whose claims we were testing.

\section{Results and Discussion}

The results of Experiment 5 are shown in Figure 10. The strength of the illusion was almost identical when it was formed by high-contrast luminance borders and when it was formed by isoluminant color borders. This was confirmed by a $t$ test for related groups $[t(19)=.05$, $p>.05]$. The very small $t$ value and the extreme closeness of the results mean that it is unlikely that a Type II error would be made, should one conclude that the two illusions have the same strength.

This finding contradicts Gregory's (1977) claim that form perception, and hence the strength of the Fraser il-

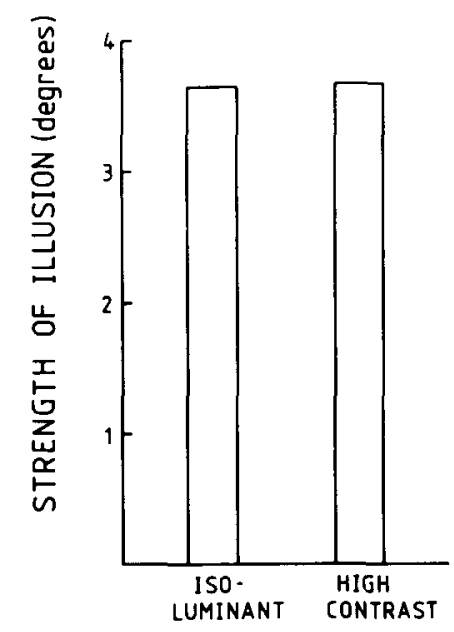

Figure 10. Results of Experiment 5, showing the strength of the illusion with background elements (as in Figure 8) when defined by high luminance contrast or isoluminant color contrast. lusion, are affected by isoluminance. It appears that in the Fraser illusion, as in the tilt aftereffect (Elsner, 1978), color contrast can be substituted for luminance contrast. Provided that most of the illusion is in the area of clear vision and-equally importantly-good color vision, the illusion persists. We note that Livingstone and Hubel (1987) have recently claimed that the Zöllner illusion, an orientation illusion thought to reflect lateral inhibition, is abolished at isoluminance. Since the tilt aftereffect is also thought to result from orientation-specific inhibition (Magnussen \& Kurtenbach, 1980), there is a theoretical disagreement that needs to be addressed by more careful measurement of both isoluminance and illusion strength. Should Livingstone and Hubel (1987) be proved correct, this would add further support to the notion that the Fraser illusion is not a result of orientation-specific interactions.

\section{GENERAL DISCUSSION}

In general, the results of the experiments on the complex figures have supported the conclusion in our previous paper that both orientation-specific interactions and the integration of information from local orientation analyzers play a role in the Fraser and related illusions. The first three experiments provided results that are difficult to explain in terms of interactions between cells with conventional receptive field structures, but quite consistent with the idea that borders are represented cooperatively by local analyzers linked by temporal synchrony of their activity.

The strength of the illusions with different types of background elements suggests that the strongest illusions result when competing local sources of orientation information are removed or reduced. Predictably, on this basis, the simple line figure with vertical edges in its directional elements showed the least illusory tilt. The figure with clubbed elements, with the vertical edges removed but with line terminators remaining, gave rise to a stronger illusion. This is difficult to explain in terms of a reduced response of cells tuned to the global orientation of the line, as was suggested by Tyler and Nakayama (1984). The strongest illusions resulted from the illusions with circular, square, or diamond background elements. These would all remove competing local orientation information, but should give rise to quite different local interactions between orientation-tuned cells, ruling out the latter process as a cause of the increased illusion.

Experiment 2 provided support for the idea that different mechanisms underlie the assimilation and contrast illusions. We have argued previously that the assimilative illusions are "direct"; that is, they result from a linking of the activity of cells responsive to the oblique edges of the directional elements. Since there would be little point to linking local analyzers with markedly different orientation preferences, the contrast illusions must be indirect, due to local orientation-specific inhibition between the oblique edges and the contour defining the edge of the twisted cord. This contour is sometimes a subjective con- 
tour, rather than one defined by luminance contrast. This does not present a problem, however, because von der Heydt and Peterhans (1989) have demonstrated directly that orientation-specific inhibition can affect cells in V2 responsive to subjective contours. Another important finding is that these cells act as local analyzers, showing length summation up to approximately $2^{\circ}$ of arc in the fovea, which is about twice that seen in V1, but is still fairly restricted in extent. This means that long subjective contours would still demand some form of cooperative coding, which has been observed to occur in Area 18 of the cat, the analogue to V2 in the primate (Eckhorn et al., 1988).

Experiment 3 is also consistent with the idea that the Fraser illusion results from the integration of information from local orientation analyzers. The simple form of the illusion showed a decrease in strength as size decreased, which seems to be the exact opposite to what would be predicted by Tyler and Nakayama (1984). At smaller visual angles where there exist cells that would be capable of coding greater and greater proportions of the overall line orientation, the illusion is progressively destroyed. It seems that if there are cells capable of coding overall orientation, they do so, free from any facilitatory influence from cells with smaller receptive fields. It is possible that cells with simple receptive field structures cannot code the overall orientation of the complex figures, and that this is consistent with the apparent dependence on local orientation information at all visual angle sizes in this case.

Recently, Morgan and Hotopf (1989) concluded that an orientation integration process must be invoked to explain the perception of diagonal contours in grids and lattices. They specifically identified this with the integration process operating in the Fraser illusion, as Morgan and Moulden (1986) did in an earlier paper on the Munsterberg illusion. In both these papers, it is suggested that integration is carried out by "collector units"-simple cells in Layer 6 of the cat visual cortex, which have large oriented receptive fields but no direct input from the lateral geniculate nucleus (Gilbert \& Wiesel, 1985). If these cells collect their input from smaller oriented units, and if there is some tolerance with respect to the orientation of the input units, a faulty coding of orientation will result.

There are some problems with this theory. Layer 6 cells project mainly to Layer 4, to the LGN and the claustrum. They seem to be part of a feedback loop, rather than an ascending pathway (van Essen, 1985). It has been suggested that their function is to confer the property of endstopping on Layer 4 cells. Bolz and Gilbert (1986) and Murphy and Sillito (1987) have shown that selective suppression or ablation of cells in Layer 6 reduces end inhibition in Layer 4 cells. These findings create difficulties for the argument that Layer 6 cells function to code global orientation. If these cells were in some higher visual area such as V2, the model would be more viable, particularly since V2 cells can respond to borders other than those defined simply by luminance contrast. However, this would make the model almost indistinguishable from that of Tyler and Nakayama (1984), who did not specify a locus for size-specific interactions. In any case, apart from the current lack of definitive physiological evidence for such mechanisms, there is a conceptual problem associated with this approach. As borders become longer and more complex, the number of higher order units required to represent them increases exponentially. At some point, it becomes necessary to invoke cooperative coding, and there is now clear evidence that this begins in the primary visual cortex.

Experiments 4 and 5, on the effects of luminance contrast and isoluminance on the Fraser illusion, have indicated some role for the processes of figure-ground segregation in the illusion. It has already been argued that these processes need to operate in both forms of the illusion in order for there to be perceived an "object" (the "twisted cord"), rather than a disconnected series of directional elements with no overall form. This object has the ecologically rare characteristic of having a different overall orientation from that of its parts.

There is some indication that the strength of the illusion may be a function of figure-ground segregation. First, simple inspection of the figures used in Experiment 4 indicated that "cords" in the outline figures were not as salient as those in the solid figures, and also that the low-contrast outline figures were particularly lacking in salience. There have been reports that the subjective contours in Kanizsa figures are weakened when the figures are drawn in outline form (Kanizsa, 1976), although Tyler (1977) claimed that some impression of contour remained. This might be expected, since to draw the components in outline form certainly reduces the impression of a solid white triangle overlaid on circular black elements.

However, it has also been demonstrated that these contours are affected by lowered contrast (Brussel, Stober, \& Bodinger, 1977) or by isoluminance (see, e.g., Gregory, 1977; Livingstone \& Hubel, 1987), whereas it was found that the Fraser illusion was unaffected by these changes. This may be because contrast spreading plays an important role in Kanizsa figures (Day, 1985; Parks, 1984) but not in other forms of subjective contour such as those resulting from discontinuities in line fields (Day, 1985; Ware, 1981). The Fraser illusion has some of the features of both; there is a definite impression of overlay, which seems to be reduced in the outline figures, but it is not clear what role, if any, contrast spreading plays in the perception of the "twisted cords."

In general, it can be said that there are two processes operating in the formation of the illusions examined. These are, first, lateral inhibitory processes that serve to improve orientation acuity yet produce contrast illusions as a side effect. Second, there is an integration process, which is based on the linking of local orientation signals to cooperatively represent the orientation (or shape) of lines longer than the receptive field size of orientation-selective cells. The best candidate for this mechanism is the phase locking of the activity of local units reported by Gray et al. (1989) and Eckhorn et al. (1988). Given such a mecha- 
nism, it is not necessary to postulate higher order cells that integrate information from local orientation units. Boundaries in an image, curved as well as straight, may be represented cooperatively by a network of neurons. Such a distributed coding scheme would be more efficient than one in which specialized cells are needed to cope with every possible combination of elementary features (Rumelhart \& McClelland, 1986). In natural scenes, it would work well and could even link together borders that are partly obscured by intervening objects. However, spurious linkages would arise from stimuli like the Fraser illusion. For these reasons, we regard it as a viable alternative to previous models involving higher order cortical cells, although at this point further research is needed before definite conclusions can be drawn.

\section{REFERENCES}

ADAM, J. (1964). A note on visual illusions of direction. Australian Journal of Psychology, 16, 53-56.

BACON, J., \& KING-SMITH, P. E. (1977). The detection of line segments. Perception, 6, 125-131.

Blakemore, C., Carpenter, R. H. S., Georgeson, M. A. (1970). Lateral inhibition between orientation detectors in the human visual system. Nature, $228,37-39$.

Bolz, J., \& Gilbert, C. D. (1986). Generation of end-inhibition in the visual cortex via interlaminar connections. Nature, 320, 362-365.

Brussel, E. M., Stober, S. R., \& Bodinger, D. M. (1977). Sensory information and subjective contours. American Journal of Psychology, 90, 145-156.

CoREN, S. (1970). Lateral inhibition and the Wundt-Hering illusion. Psychonomic Science, 18, 341.

Cowan, T. M. (1973). Some variation[s] of the twisted cord illusion and their analyses. Perception \& Psychophysics, 14, 553-564.

DAvidoff, J. (1973). Contrast effects in geometrical illusions. Vision Research, 13, 201-205.

DAY, R. H. (1985). Enhancement of edges by contrast, depth and figure: The origins of illusory contours. In J. D. Pettigrew, K. Sanderson, \& W. R. Levick (Eds.), Visual neurosciences (pp. 352-364). Cambridge, U.K.: Cambridge University Press.

Dow, B. M., SNyder, A. Z., VAutin, R. G., \& Bauer, R. (1981). Magnification factor and receptive field size in foveal striate cortex of the monkey. Experimental Brain Research, 44, 213-228.

ECKhorn, R., Bauer, R., Jordan, W., Brosch, M., Kruse, W., MunK, M., \& ReItboek, H. J. (1988). Coherent oscillations: A mechanism of feature linking in the visual cortex? Biological Cybernetics, 60, 121-130.

ELSNER, A. (1978). Hue difference contours can be used in processing orientation information. Perception \& Psychophysics, 24, 451-456.

Fraser, J. (1908). A new visual illusion of direction. British Journal of Psychology, 2, 307-320.

GILBERT, C. D., \& WIESEL, T. N. (1985). Intrinsic connectivity and receptive field properties in visual cortex. Vision Research, 25, 365-374.

Gray, C. M., Konig, P., Engel, A. K., \& Singer, W. (1989). Oscillatory responses in cat visual cortex exhibit inter-columnar synchronization which reflects global stimulus properties. Nature, 338, 334-337.

GREGORY, R. L. (1977). Vision with isoluminant colour contrast: 1. A projection technique and observations. Perception, 6, 113-119.

How ARD, I. P. (1982). Human visual orientation. New York: Wiley.

JUDD, D. B. (1951). Basic correlates of the visual stimulus. In S. S. Stevens (Ed.), Handbook of experimental psychology (pp. 811867). New York: Wiley.

KanIzsA, G. (1976). Subjective contours. Scientific American, 234, 48-52.
Livingstone, M. S., \& Hubel, D. H. (1987). Psychophysical evidence for separate channels for the perception of form, color, movement and depth. Journal of Neuroscience, 7, 3416-3468.

MAGNusSeN, S., \& KURTENBACH, W. (1980). Linear summation of tilt illusion and tilt aftereffect. Vision Research, 20, 39-42.

MoRgan, M. J., \& Hotopf, W. H. N. (1989). Perceived diagonals in grids and lattices. Vision Research, 29, 1005-1015.

Morgan, M. J., Moulden, B. (1986). The Munsterberg figure and twisted cords. Vision Research, 26, 1793-1800.

MurPhy, P. C., \& Sillito, A. M. (1987). Corticofugal feedback influences the generation of length tuning in the visual pathway. $\mathrm{Na}$ ture, 329, 727-729.

O'TOOLE, B. I. (1979). The tilt illusion: Length and luminance changes of induction line and third (disinhibiting) line. Perception \& Psychophysics, 25, 487-496.

O'TOOLE, B. [I.], \& Wenderoth, P. (1977). The tilt illusion: Repulsion and attraction effects in the oblique meridian. Vision Research, 17, 367-374.

Oyama, T. (1975). Determinants of the Zöllner illusion. Psychological Research, 37, 261-280.

PARKER, D. M. (1974). Evidence for the inhibition hypothesis in expanded angle illusion. Nature, 250, 265-266.

PARKs, T. E. (1984). Illusory figures: A (mostly) atheoretical review. Psychological Bulletin, 95, 282-300.

Peterhans, E., von der Heydt, R. (in press). The whole and the pieces-Cortical neuron responses to bars and rows of moving dots. In J. J. Kulikowski, C. M. Dickinson, \& I. J. Murray (Eds.), Seeing contour and colour: Proceedings of the Third International Symposium of the Northern Eye Institute, Manchester, U.K. 9-13 August, 1987. New York: Pergamon Press.

Poggio, G. F. (1972). Spatial properties of neurons in striate cortex of unanaesthetised monkey. Investigative Ophthalmology, 11, 368-387.

RoBinson, J. O. (1972). The psychology of visual illusion. London: Hutchinson.

Rumelhart, D. E., \& MCClelland, J. L. (1986). Parallel distributed processing: Explorations in the microstructure of cognition: Vol. 1. Foundations. Cambridge, MA: MIT Press.

SMITH, A. T., \& OVER, R. (1977). Orientation masking and the tilt illusion with subjective contours. Perception, 6, 441-447.

Stuart, G. W., \&Ay, R. H. (1980). The effect of blurring on intersecting-line illusions. Perception, 9, 537-548.

StuART, G. W., \& DAY, R. H. (1988). The Fraser illusion: Simple figures. Perception \& Psychophysics, 44, 409-420.

ThомAs, J. P. (1978). Spatial summation in the fovea: Asymmetrical effects of longer and shorter dimensions. Vision Research, 18, 1023-1029.

TYLER, C. W. (1977). Is the illusory triangle physical or imaginary? Perception, 6, 603-604.

TyLER, C. [W.], NAKAYAMA, K. (1984). Size interactions in the perception of orientation. In L. Spillman \& J. R. Wooten (Eds.), Sensory experience, adaptation and perception (pp. 529-546). New York: Erlbaum.

VAN ESSEN, D. C. (1985). Functional organization of primate visual cortex. In A. Peters \& E. G. Jones (Eds.), Cerebral cortex (Vol. 3, pp. 259-329). New York: Plenum.

von der Heydt, R., \& Peterhans, E. (1989). Mechanisms of contour perception in monkey visual cortex: I. Lines of pattern discontinuity. Journal of Neuroscience, 9, 1731-1748.

von der Heydt, R., Peterhans, E., \& Baumgartner, G. (1984). Illusory contours and cortical neuron responses. Science, 224, $1260-1262$

WALLACE, G. K. (1975). The effect of contrast on the Zöllner illusion. Vision Research, 15, 963-966.

WARE, C. (1981). Subjective contours independent of subjective brightness. Perception \& Psychophysics, 29, 500-504.

(Manuscript received March 20, 1989; revision accepted for publication November 28, 1990.) 